\title{
JUSTUS LLEBIG'S
}

\section{ANNALEN DER CHEMIE.}

248. B a nd.

Mittheilungen aus dem ehemisehen Laboratorinm von Prof. Dr. J. W i slicenus in Leipzig.

(Eingelaufen den 5. August 1888.)

101. Untersuchungen zur Bestimmung der räumlichen Atomlagerung; von Johannes Wislicenus. D r i t t e A b ha nd l u n g. Ueber einige Glieder der Stilbengruppe; von A. Blank.

Vom Benzoïn, dem am leichtesten zu gewinnenden Gliede der Stilbengruppe ausgehend, ist durch Einwirkung der verschiedensten Reagentien eine Reihe von Verbindungen erhalten worden, welche zum $Z$ wecke der Erforschung ihrer Structur Anlafs zu umfangreichen Arbeiten gaben. Namentlich ist der Einflufs reducirender Mittel auf das Benzoin $\mathrm{C}_{14} \mathrm{H}_{12} \mathrm{O}_{2}$ vielfach studirt worden. Die so erhaltenen Körper sind entweder Producte der Wasserstoffaddition, oder der Sauerstoffentziehung, oder verdanken beiden Vorgängen, welche mit einander verlaufen, ihre Entstehung.

Durch Einwirkung von Zink und Salzsäure auf eine alkoholische Benzoünlösung erhielt $\mathrm{Zinin*)}$ ein desoxydirtes Benzoïn $\mathrm{C}_{14} \mathrm{H}_{12} \mathrm{O}$, Grima u $\mathrm{x}^{* *}$ ) bei Anwendung von Natrium-

*) Ann. Chem. $\mathbf{1 2 0}, 218$.

**) Ber. d. deutsch, chem. Ges. 2, 281. 
amalgam als Wasserstoffquelle dagegen ein hydrogenisirtes, das Hydrobenzoïn $\mathrm{C}_{14} \mathrm{H}_{14} \mathrm{O}_{2}$.

Goldenberg*) liefs Natriumamalgam auf eine stets schwach sauer gehaltene Benzoïnlösung wirken und fand neben Hydrobenzoïn und Desoxybenzoïn ein bei $208^{\circ}$ schmelzendes Benzoïnpinakon $\mathrm{C}_{28} \mathrm{H}_{26} \mathrm{O}_{4}$. Die Entstehung dieser Verbindung kounte indefs $\mathrm{Zagoumenny} \mathrm{y}^{*}$ ) nicht beobachten, er erhielt immer nur eine Verbindung $\mathrm{C}_{28} \mathrm{H}_{26} \mathrm{O}_{2}$, die früher schon Limpricht und Schwanert***) durch Reduction des Desoxybenzoïns dargestellt hatten.

Bezüglich der Constitution dieser Verbindungen waren die Meinungen sehr getheilt. Grimauxt), sowie Limpricht und Schwanert $+f$ ), später auch Kekulé $+t+$ ) leiteten dieselben von einem symmetrischen Diphenyläthylen ab :

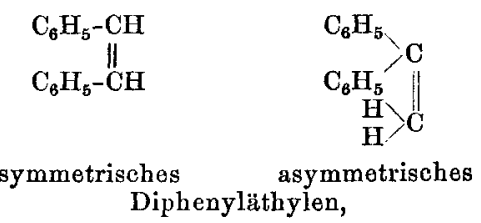

während S tädeler *), vom unsymmetrischen Diphenyläthylen ausgehend, die Structurformeln jener Derivate entwickelte. Bei Besprechung der Constitution einiger Reductionsproducte des Desoxybenzoïns gelangten jedoch Limpricht und Schwanert ${ }^{*}$ ), gestützt auf die nahen Beziehungen, welche

*) Ann. Chem. 184, 332 .

**) Ber. d. deutsch. chem. Ges. E, 1651.

***) Ann. Chem. 155, 62.

†) Bull. soc. chim. 1867, $\mathrm{T}$ ₹, 378.

††) Ann. Chem. 145, 330.

††) Ber. d. deutsch. chem. Ges. 2, 611.

*) Ann. Chem. 151,44 bis 46 .

**) Daselbst 155, 75 . 
zwischen Benzil und Benzilsäure herschen, zu der Ansicht, dafs die $S$ tädeler'schen Formeln die richtigen seien. Sie schrieben deshalb dem Desoxybenzoïn und der daraus durch Wasserstoffaddition neben Toluylenhydrat entstehenden Verbindung $\mathrm{C}_{28} \mathrm{H}_{26} \mathrm{O}_{2}$ vom Schmelzpunkt $156^{0}$ folgende Structurformeln zu :

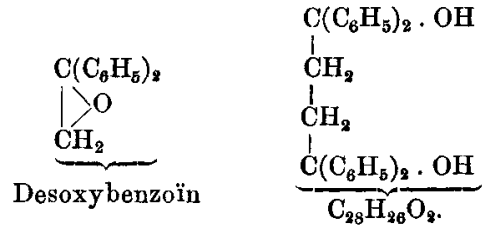

$\mathrm{Zag} \mathrm{ou} \mathrm{m} \mathrm{e} \mathrm{n} \mathrm{n} \mathrm{y}{ }^{*}$ ) stellte den bei $156^{\circ}$ sich verflüssigenden Körper durch Einwirkung von Zink und alkoholischer Kalilauge auf Desoxybenzoïn dar, und es gelang ihm, denselben im reinen Zustande durch häufig wiederholtes Umkrystallisiren zu gewinnen. Das gereinigte Product schmolz bei $213^{\circ}$.

Beim Erhitzen von Benzoïn mit alkoholischem Natriumäthylat in zugeschmolzenen Röhren erhielten $\mathrm{Jen}$ a und Limpricht**) eine ebenfalls nach der Formel $\mathrm{C}_{28} \mathrm{H}_{26} \mathrm{O}_{2}$ zusammengesetzte Verbindung, deren Schmelzpunkt bei $61^{\circ}$ liegt. Sie sind geneigt, derselben eine ähnliche asymmetrische Constitution zuzuschreiben, wie dem von Limpricht und Schwanert aus Desoxybenzoïn dargestellten Körper $\mathrm{C}_{28} \mathrm{H}_{26} \mathrm{O}_{2}$. Kurz darauf gelangte $\mathrm{Z}$ inck $\mathrm{e}^{\text {****; }}$ ), indem er allen bekannten Thatsachen Rechnung trug, auf Grund seiner Versuche zu dem Schlufs, dafs den Stilbenderivaten dennoch eine symmetrische Structur zukomme. In der Folge erfuhr diese Anschauung durch die Resultate anderer Forscher volle Bestätigung. Die Auffindung des vom Stilben vollkommen ver-

*) Ber. d. deutsch. chem. Ges. 5, 1102.

**) Ann. Chem. 155, 96 .

***) Ber. d. deutsch. chem. Ges. 4, 837. 
schiedenen asymmetrischen Diphenyläthylens durch $\mathrm{Hepp}{ }^{*}$ ) bewies indirect, aber endgültig, dafs die Stilbenverbindungen die beiden Phenylgruppen an verschiedene Kohlenstoffatome gebunden enthalten.

Das Verdienst, zuerst die relative Stellung der Sauerstoffatome in diesen Verbindungen bestimmt zu haben, kommt Radziszewski**) zu. Derselbe stellte Desoxybenzoïn synthetisch dar durch trockene Destillation eines Gemisches von benzoësaurem und phenylessigsaurem Calcium, und er sprach es daher als ein Keton an. Erst später wiesen Victor Meyer und Goldschmidt $* * *$ ) die Ketongruppen im Benzoïn und Benzil nach. Weil nun aus einem Keton durch Aufnahme von Wasserstoff ein Pinakon entsteht, bezeichnete $R$ adziszewski den von Limpricht und $S c h w a n e r t$ bei der Reduction des Desoxybenzoïns erhaltenen Körper $\mathrm{C}_{28} \mathrm{H}_{26} \mathrm{O}_{2}$ als Desoxybenzoïnpinakon. Merkwürdigerweise findet sich im Handbuch der organischen Chemie von Beilstein (2. Auflage, Bd. II, 708) für dieses Pinakon die Formel :

$$
\begin{aligned}
& \mathrm{C}_{6} \mathrm{H}_{5}-\mathrm{CH}(\mathrm{OH}) . \mathrm{CHC}_{6} \mathrm{H}_{5} \\
& \mathrm{C}_{6} \mathrm{H}_{5}-\mathrm{CH}(\mathrm{OH}) . \mathrm{CHC}_{6} \mathrm{H}_{5},
\end{aligned}
$$

welche mit der Entstehung des Körpers im Widerspruche steht und welche überhaupt kein Pinakon vorstellt, da ein solches die Hydroxylgruppen an benachbarte Kohlenstoffatome gebunden enthalten mufs. Wahrscheinlich ist diese Formel der von Limpricht und $\mathrm{Schwanert}$ zuerst gegebenen nachgebildet, indem in derselben, gemäls den späteren Ansichten, die Phenylgruppen symmetrisch am Kern vertheilt wurden.

*) Ber. d. deutsch. chem. Ges. 8, 1109.

**) Daselbst 6, 491 und 8, 756.

***) Daselbst 16, 504 und 2176 
Nach Zagoumenny's Untersuchungen mufste es als unzweifelhaft erscheinen, dafs dieses von Limpricht und Schwanert dargestellte Pinakon vom Schmelzpunkt $156^{\circ}$ im reinen Zustande bei $213^{\circ}$ schmilzt. Im Laboratorium des Herrn Professor Wislicenus wurden gelegentlich der Darstellung von Desoxybenzoin aus Benzoin mit Eisessig und Zinkstaub als Nebenproducte aufser diesem Körper vom Schmelzpunkte $213^{0}$ noch Substanzen erhalten, welche bedeutend niedriger, zwischen 155 und $160^{\circ}$ schmolzen. Dieselben konnten kein Gemenge des hochschmelzenden Pinakons mit den schon bekannten Reductionsproducten des Benzoins : „Desoxybenzoin, Hydrobenzoin und Toluylenhydrat" sein, da die letzteren, als sehr leicht in Alkohol löslich, nicht wohl in den öfters umkrystallisirten, schwer löslichen, bei 155 bis $160^{\circ}$ sich verflüssigenden Substanzen enthalten sein konnten. Es mufste sich sonach ein anderes, noch nicht charakterisirtes Product mitgebildet haben, und es gelang in der That, dasselbe zu fassen.

\section{Reduction des Benzoïns.}

Um einen Einblick in den Verlauf des Reductionsprocesses des Benzoïns zu bekommen, wurden in mehreren Versuchen die entstandenen Producte nach verschiedener Dauer der Wasserstoffeinwirkung isolirt und annähernd quantitativ bestimmt.

Eine Lösung von $60 \mathrm{~g}$ Benzoïn in $400 \mathrm{~g}$ Eisessig wurde auf dem Wasserbade erhitzt und in dieselbe während eines Zeitraumes von 2 Stunden nach und nach $120 \mathrm{~g}$ Zinkstaub eingetragen. Das Erhitzen wurde beim 1. Versuch noch 8, beim zweiten 14, beim dritten 19 Stunden fortgesetzt. Durch Hinzufügen von etwa 80 bis $100 \mathrm{~g}$ warmen Wassers zur Reactionsmasse löste sich der gröfste Theil des ausgeschiedenen Zinkacetals wieder auf. Die fast klare Lösung wurde vom 
Zinkstaub abgegossen, der letztere auf einem Filter gesammelt und mit wenig Eisessig ausgelaugt. Filtrat und Lösung liefsen beim Verdünnen mit viel kaltem Wasser eine Krystallmasse, welcher 0el anhaftete, fallen. Dieselbe wurde abfiltrirt und ausgewaschen, das Filtrat mit Soda fast neutralisirt und mit Aether ausgeschüttelt. Nach dem Verdunsten des letzteren blieb ein Oel zurück, das beim langen Stehen theilweise erstarrte und welches man am besten zusammen mit der durch Wasser ausgeschiedenen Krystallmasse verarbeitet.

Der abfiltrirte und mit etwas Eisessig ausgewaschene, unangegriffene Zinkstaub wird am besten mit kochendem Benzol bis zur Erschöpfung ausgezogen. Nach dem Verdunsten des Lösungsmittels hinterbleiben fast weifse, nadelförmige Krystalle, welche bei $200^{\circ}$ schmelzen. Durch Umkrystallisiren wird der Schmelzpunkt derselben auf $213^{\circ}$ erhöht. Eine Analyse ergab folgende Zahlen :

I. $0,1830 \mathrm{~g}$ Substanz lieferten $0,5704 \mathrm{CO}_{2}$ und $0,1109 \mathrm{H}_{\mathbf{z}} \mathrm{O}$.

II. $0,2035 \mathrm{~g} n$ n $0,6370 n \pi 0,1245$ n

\begin{tabular}{ccrc} 
& $\begin{array}{c}\text { Berechnet für } \\
\mathrm{C}_{28} \mathrm{H}_{26} \mathrm{O}_{\mathbf{2}}\end{array}$ & \multicolumn{2}{c}{ Gefunden } \\
$\mathrm{C}$ & 85,28 & $\mathrm{I}$. & $\mathrm{II}$. \\
$\mathrm{H}$ & 6,60 & 65,01 & 85,37 \\
& 6,73 & $6,78$.
\end{tabular}

Sonach war diese Substanz identisch mit dem Desoxybenzoinpinakon, welches $\mathrm{Zag}$ oumenny zuerst rein dargestellt hatte.

Die aus dem Filtrate vom Zinkstaub erhaltene, mit Oel behaftete Krystallmasse wird, wie sich im Laufe der Untersuchung ergab, am besten in folgender Weise behandelt. Man versetzt dieselbe mit nicht zu wenig Aether und schüttelt gut um. Den ungelösten Theil sammelt man auf einem Filter und wäscht ihn so lange mit Aether aus, bis er über $200^{\circ}$ schmilzt. Er ist im wesentlichen das obenerwähnte Desoxybenzoünpinakon. Aus dem ätherischen Filtrate setzen sich im Verlaufe einiger Tage viele kleine nadelförmige Krystalle 
$a b$, welche nach dem Verdünnen der syrupdicken Masse mit Aether auf einem Filter gesammelt und mit wenig Aether ausgewaschen werden. Dieselben schmelzen bei $156^{\circ}$. Das Filtrat in der gleichen Weise behandelt, gab noch eine geringe Menge dieser Nadeln. Die restirende dichflüssige Masse mit wenig starkem Alkohol versetzt, erstarrte zum Theil und die ausgeschiedenen Krystalle liefsen sich beim Umkrystallisiren mit Leichtigkeit in zwei Substanzen trennen, von denen die eine schwerer lösliche bei $124^{\circ}$, die leichter lösliche bei $56^{\circ}$ schmolz. Die erstere tritt in geringerer Menge auf.

Doch verblieb in den Mutterlaugen immer noch eine nicht unbedeutende Menge eines Oeles, das einen angenehmen hyacinthenähnlichen Geruch besafs. Dasselbe wurde der Destillation unterworfen. Anfangs ging eine Spur Wasser und Essigsäure über und erst bei ungefähr $280^{\circ} \mathrm{kam}$ die Flüssigkeit in lebhaftes Sieden. Die Hauptmasse destillirte zwischen 300 und $325^{\circ}$ über. Im Kölbchen blieb noch ein dunkler öliger Rückstand. Das anfangs flüssige Destillat erstarrte beim Stehen zum gröfsten Theile, und nach dem Umkrystallisiren konnten wieder die obengenannten beiden bei $124^{\circ}$ und $56^{\circ}$ schmelzenden Substanzen erhalten werden. Der erstere, höher schmelzende Körper krystallisirt in prachtvoll perlmutterglänzenden Blättchen und erwies sich bei der Analyse als Stilben:

$0,1879 \mathrm{~g}$ Substanz gaben $0,6429 \mathrm{CO}_{\mathbf{2}}$ und $0,1168 \mathrm{H}_{\mathbf{8}} \mathrm{O}$.

$\begin{array}{ccc} & \text { Berechnet für } & \text { Gefunden } \\ \mathrm{C} & \mathrm{C}_{14} \mathrm{H}_{12} & \\ \mathrm{H} & 93,33 & 93,31 \\ & 6,67 & 6,90 .\end{array}$

Die bei $56^{\circ}$ schmelzende Verbindung besafs alle Eigenschaften und die procentische Zusammensetzung des Desoxybenzoïns :

$0,1905 \mathrm{~g}$ Substanz lieferten $0,5985 \mathrm{CO}_{2}$ und $0,104 \mathrm{H}_{2} \mathrm{O}$. 


$\begin{array}{ccc} & \text { Berechnet für } & \text { Gefunden } \\ & \mathrm{C}_{14} \mathrm{H}_{12} \mathrm{O} & \\ \mathrm{C} & 85,72 & 85,69 \\ \mathrm{H} & 6,12 & 6,07 .\end{array}$

Die letzten Mutterlaugen gaben beim Verdunsten des Alkohols noch etwas 0el, welches keinen constanten Siedepunkt zeigte. Aus dem Destillate schieden sich wiederum Stilben und Desoxybenzoin unter Hinterlassung eines nicht mehr erstarrenden 0eles ab. Der anfangs erhaltene Syrup besteht sonach zumeist aus den beiden zuletzt genannten Körpern, welche durch die Gegenwart einiger öliger Substanzen, unter denen sich vielleicht das Acetat des Toluylenhydrates, sowie eine hyacinthenähnlich riechende Flüssigkeit befinden, dickflüssig erhalten werden.

In nachstehender Tabelle sind die aus $60 \mathrm{~g}$ Benzoïn bei 10-, 16- und 21stündiger Reduction gewonnenen Producte ihrer Menge nach zusammengestellt:

I.

\begin{tabular}{|c|c|c|c|}
\hline & $\begin{array}{c}10 \text { stündige } \\
\text { Reduction }\end{array}$ & $\begin{array}{l}16 \text { stündige } \\
\text { Reduction }\end{array}$ & $\begin{array}{l}21 \text { stündige } \\
\text { Reduction }\end{array}$ \\
\hline $\begin{array}{l}\text { Desoxybenzoinpinakon, } \\
\text { Schmelzpunkt } 213^{0}\end{array}$ & 10,6 & 13,2 & 14,0 \\
\hline $\begin{array}{l}\text { Nadeln, Sehmelzpunkt } \\
155 \text { bis } 160^{\circ}\end{array}$ & 3,1 & 6,6 & 11,0 \\
\hline Stilben, Schmelzpunkt $124^{\circ}$ & 2,0 & 2,0 & 2,4 \\
\hline $\begin{array}{c}\text { Desoxybenzoïn, Schmelz- } \\
\text { punkt } 56^{\circ}\end{array}$ & 30,0 & 24,0 & 18,0 \\
\hline Zuletzt überbleibendes Oel & 4,0 & 3,0 & 3,0 \\
\hline
\end{tabular}

II. III.
Es zeigte sich, dafs schon nach 3 stündiger Reaction alles Benzoïn in Desoxybenzoïn umgewandelt war. Aus der Tabelle ist ersichtlich, dafs bei weiter zunehmender Reductionsdauer die Menge des Desoxybenzoïns abnimmt, während die der übrigen Producte sich vermehrt. Auffällig ist es, dafs die fraglichen Nadeln vom Schmelzpunkt 155 bis $160^{\circ}$ mit wachsender 
Dauer der Wasserstoffeinwirkung auf das Desoxybenzoin, also bei steigender Verdünnung der Säure bis zu einem gewissen Grade in viel gröfserer Proportion zunehmen, als die bei $213^{\circ}$ schmelzenden Nadeln von Desoxybenzoinpinakon.

Untersuchung der Nadeln vom Schmelzpunkt 155 bis $160^{\circ}$.

Dieselben zeigen ähnliche Löslichkeitsverhältnisse, wie das Pinakon vom Schmelzpunkt $213^{\circ}$. Sie sind unlöslich in Wasser; in kaltem Alkohol und Aether lösen sie sich schwer, in warmen leichter, sehr reichlich dagegen in Benzol und Chloroform. Durch sehr oft wiederholtes Umkrystallisiren aus Alkohol konnte eine geringe Menge des bei $213^{\circ}$ schmelzenden Pinakons daraus erhalten werden. Die aus den einzelnen Filtraten ausgeschiedenen Nadeln schmolzen bei verschiedenen Temperaturen, aber alle zwischen 156 und $170^{\circ}$, und es gelang nicht, auf diese Weise ein constant schmelzendes Product zu bekommen. Ebenso eignen sich die anderen angeführten Lösungsmittel nicht zur Gewinnung einer einheitlichen Substanz. Man gelangt jedoch bei Anwendung von Aceton zum Ziele. In einem Becherkolben mit engem Halse oder einer mit Glasplatte bedeckten Krystallisirschale löst man die bei 156 bis $170^{\circ}$ schmelzenden Nadeln in nicht zu wenig warmen Acetons und läfst die Schale oder das Kölbchen ruhig mehrere Tage stehen. Das Aceton verdunstet langsam und es scheiden sich aus der Flüssigkeit Krystalle verschiedener Form $a b$ :

1) warzenförmig gruppirte oder büschelförmig vereinigte feine Nadeln,

2) langgestreckte kleine Blättchen, aus welchen bei zunehmender Dicke Prismen werden, die sich einzeln oder auch zu Gruppen vereinigt ausbilden.

Durch Auslesen lassen sich die beiden Substanzen von einander leicht trennen. Die Blättchen oder Prismen besitzen 
den Schmelzpunkt 208 bis $210^{\circ}$, nach nochmaligem Umkrystallisiren verflüssigen sie sich bei $213^{\circ}$ und erweisen sich als das schon bekannte und oben beschriebene Desoxybenzoïnpinakon.

Die feimen Nadeln, welche warzen- oder büschelförmig vereinigt sind, schmolzen bei $168^{\circ}$. Durch wiederholtes Umkrystallisiren aus Aceton und Alkohol erhält man ein constant bei $172^{\circ}$ schmelzendes Product. Dasselbe krystallisirt aus allen Lösungsmitteln in Nadeln, niemals in Blättchen. In kaltem Alkohol ist es schwer löslich. $100 \mathrm{~g}$ desselben lösen etwa 0,7 bis 0,8 Th. dieses Körpers. Bei der Analyse gab er Zahlen, welche auf die Formel $\mathrm{C}_{28} \mathrm{H}_{26} \mathrm{O}_{2}$ stimmen.

I. $0,2076 \mathrm{~g}$ Substanz gaben $0,6484 \mathrm{CO}_{2}$ und $0,1235 \mathrm{H}_{2} \mathrm{O}$.

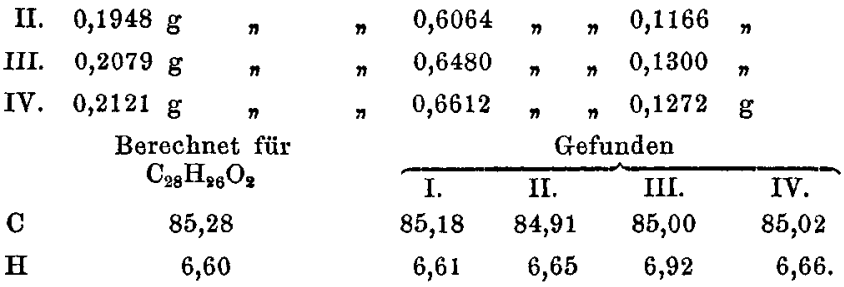

Merkwürdigerweise besitzt sonach diese bei $172^{\circ}$ schmelzende Substanz dieselbe Zusammensetzung wie das bei $213^{\circ}$ sich verflüssigende Desoxybenzoïnpinakon. Ihrer Entstehung und ihren Eigenschaften zufolge mufs sie ebenfalls als ein Pinakon des Phenylbenzylketons angesehen werden. Die Formel :

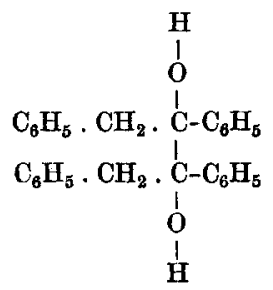

kommt sonach beiden Pinakonen zu. Die Atomgruppirung : 


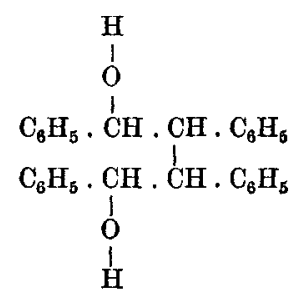

ist ausgeschlossen, da beide Pinakone durch Reduction des Desoxybenzoïns mit Essigsäure und Zinkstaub neben einander entstehen, wie dies aus der oben angeführten Tabelle ersichtlich ist. Doch wurde mit reinem Phenylbenzylketon der Versuch wiederholt. In eine Lösung von $15 \mathrm{~g}$ Desoxybenzoïn in $100 \mathrm{~g}$ Eisessig und $15 \mathrm{~g}$ Wasser wurden $15 \mathrm{~g}$ Zinkstaub allmählich eingetragen und dieselbe 10 Stunden lang auf dem Wasserbade erhitzt. Die Reductionsproducte wurden in ähnlicher Weise getrennt wie die des Benzoinns, und es konnte unter ihnen mit voller Sicherheit sowohl das bei $213^{\circ}$, als auch das bei $172^{\circ}$ schmelzende Pinakon nachgewiesen werden.

Ein Gemisch beider verflüssigt sich bei 156 bis $160^{\circ}$.

Es sei die bei $213^{0}$ schmeizende, in blättchenähnlichen Nadeln oder Prismen krystallisirende Verbindung als $\alpha$-Desoxybenzoïninakon bezeichnet, während der Name $\beta$-Desoxybenzoïnpinakon den bei $172^{\circ}$ in den flüssigen Zustand übergehenden Nadeln zukomme.

Verhalten der Desoxybenzö̈npinakone beim Erhitzen.

$\mathrm{Zag}$ o u menny*) fand, dafs sich das $\alpha$-Desoxybenzoïnpinakon beim Destilliren in Desoxybenzoin und Toluylenhydrat zersetzt. Eine Wiederholung des Versuches ergab dasselbe Resultat.

Erhitzt man $\beta$-Desoxybenzoüninakon auf $300^{\circ}$, oder destillirt man es, so erleidet es genau dieselbe Zersetzung.

*) Ber. d. dentsch, chem. Ges. 5, 1102. 
Das farblose ölige Destillat mit Alkohol versetzt schied bald Blättchen aus, welche nach dem Umkrystallisiren bei $56^{0}$ schmolzen und die Eigenschaften und Zusammensetzung des Desoxybenzoïns hatten :

$0,1600 \mathrm{~g}$ Substanz gaben $0,5037 \mathrm{CO}_{2}$ und $0,0877 \mathrm{H}_{2} \mathrm{O}$, entsprechend 85,81 pC. C und 6,08 pC. $\mathrm{H}$.

Berechnet für $\mathrm{C}_{14} \mathrm{H}_{12} \mathrm{O} 85,72$ pC. $\mathrm{C}$ und 6,12 pC. $\mathrm{H}$.

Aus dem Filtrate erhielt ich durch mehrfach wiederholtes Umkrystallisiren bei $42^{\circ}$ schmelzende Nadeln, welche ihrem äufseren Ansehen, den Löslichkeitsverhältnissen und dem Schmelzpunkte nach Toluylenhydrat waren. Es gelang nicht, dieselben vollkommen frei von Desoxybenzoïn zu gewinnen. Auf eine Elementaranalyse wurde daher verzichtet und der Identitätsnachweis auf folgende Art geführt : Die Nadeln wurden mit verdünnter Schwefelsäure gekocht. Die in der Flüssigkeit herumschwimmenden Oeltröpfchen erstarrten beim Erkalten zu Blättchen, welche nach dem Umkrystallisiren bei $124^{0}$ schmolzen und das Aussehen von Stilben hatten. In Aether gelöst und mit Brom versetzt fiel bei $235^{\circ}$ schmelzendes Stilbendibromid aus, so dafs kein $\mathrm{Z}_{w}$ eifel an der Identität der Nadeln mit Toluylenhydrat herrschen konnte. Die Zersetzung war sonach, der Erwartung entsprechend, nach folgender Gleichung verlaufen :

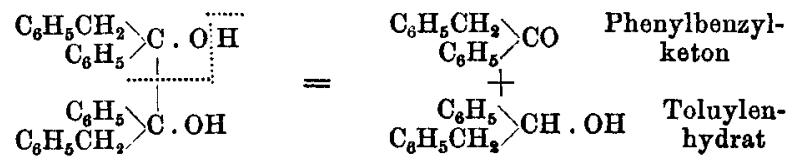

Desoxybeuzoïnpinakon

also genau in derselben Weise wie die eines jeden Pinakons.

Die Reduction des Benzoïns in Eisessiglösung verläuft mithin in ähnlichem Sinne wie die in anderen sauer gehaltenen Lösungen. Es wird zuerst die Alkoholgruppe von dem entwickelten Wasserstoff angegriffen, und es entsteht Phenylbenzylketon : 


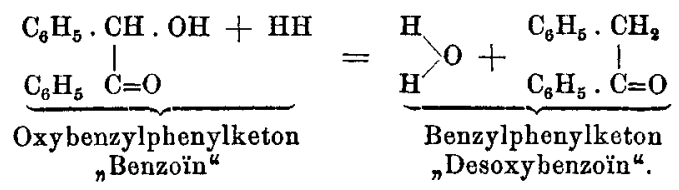

In alkalischer Lösung verwandelt dagegen der Wasserstoff im status nascendi zunächst die Ketongruppe in eine Alkoholgruppe, indem sich Hydrobenzoïn bildet :

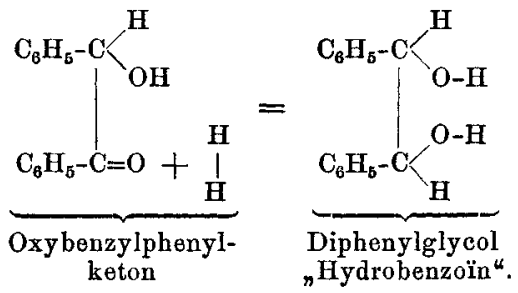

Das Desoxybenzoin wird als Keton bei der weiteren Reduction in den entsprechenden secundären Alkohol, das Toluylenhydrat übergehen :

$$
\underbrace{\mathrm{C}_{6} \mathrm{H}_{5} \cdot \mathrm{C}_{6} \mathrm{H}_{5} \cdot \mathrm{CH}_{2}}_{\begin{array}{c}
\text { Phenylbenzyl- } \\
\text { keton }
\end{array}}=\underbrace{\mathrm{C}_{6} \mathrm{H}_{5} \cdot \mathrm{C}_{\mathrm{OH}}^{\mathrm{H}}}_{\begin{array}{c}
\text { Phenylbenzyl- } \\
\text { carbinol } \\
{ }^{\text {Tholuylenhydrat }}{ }^{\mu},
\end{array}}
$$

wenn sich viel und schnell Wasserstoff in der Lösung entwickelt. Da jedoch im gegebenen Falle die Wasserstoffbildung nur in den ersten Stunden eine lebhaftere ist, später aber abnimmt, weil das gebildete Zinkacetat die Einwirkung der Essigsäure auf den Zinkstaub hemmt, so sind nach Umwandlung des Benzoïns in Phenylbenzylketon alle Bedingungen zu einer möglichst ausgiebigen Pinakonbildung gegeben. Thatsächlich entsteht ja neben viel Pinakon nur sehr wenig Toluylenhydrat, welches jedenfalls durch die Essigsäure *) dieselbe Zersetzung wie durch verdünnte Schwefelsäure er-

*) Vielleicht unterstützt durch die Anwosenheit von Zinkacetat. 
leidet, nämlich in Wasser und Stilben gespalten wird, entsprechend der folgenden Gleichung :

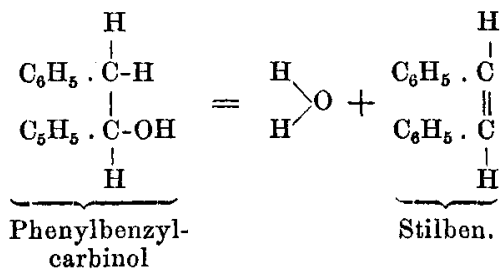

Dadurch wird die Entstehung dieses ungesättigten Kohlenwasserstoffes in der Wasserstoff entwickelnden Flüssigkeit verständlich. Dibenzyl und Hydrobenzoïn konnten nicht unter den Reductionsproducten nachgewiesen werden.

Die Ausbeute an Stilben mufste zunehmen, wenn man durch Wasserzusatz oder Anwendung einer verdünnteren Säure dafür sorgte, dafs das Zinkacetat möglichst in Lösung gehalten und dadurch eine intensivere Gasentwickelung erzielt wurde. In der That ergaben sich bei Anwendung einer 50 procentigen Säure und 25 stündiger Reduction folgende Zahlen :

$60 \mathrm{~g}$ Benzoïn lieferten 8,7 g $\alpha$-Desoxybenzoïnpinakon.

$7,8 \mathrm{~g}$ des Gemisches von $\alpha$-und $\beta$-Desoxybenzoïnpinakon vom Schmelzpunkt 155 bis $160^{\circ}$.

$8,2 \mathrm{~g}$ Stilben.

15,0 g Desoxybenzoïn.

$4,0 \mathrm{~g}$ Oel und

$1,8 \mathrm{~g}$ Hydrobenzoïn.

Die Verarbeitung und Trennung der Producte geschah in der früher angegebenen Weise. Der ätherische Auszug des wässerigen, das Zinkacetat enthaltenden Filtrates hinterliefs nach dem Verdunsten des Aethers eine Substanz, welche nach dem Umkrystallisiren bei $134^{\circ}$ schmolz. Sie bildete weifse, glänzende, leichte Blättchen und besafs alle Eigenschaften und die Zusammensetzung des Hydrobenzoïns. 
$0,1980 \mathrm{~g}$ Substanz gaben 0,5720 $\mathrm{CO}_{2}$ und $0,1109 \mathrm{H}_{8} \mathrm{O}$.

$\begin{array}{ccc} & \text { Berechnet für } & \text { Gefunden } \\ & \mathrm{C}_{14} \mathrm{H}_{14} \mathrm{O}_{2} & \\ \mathrm{C} & 78,51 & \mathbf{7 8 , 7 8} \\ \mathrm{H} & 6,54 & 6,22 .\end{array}$

Eine Spur Desoxybenzoïn, von der die geringe Menge Hydrobenzoïn nicht mehr befreit werden konnte, verursachte die kleinen Abweichungen in den Procentzablen.

Es wird sonach bei Anwendung von 50 procentiger Essigsäure die Ketongruppe eines allerdings nur geringen Procenttheiles des Benzoïns vor der Alkoholgruppe von dem nascirenden Wasserstoff angegriffen, ein Vorgang, der bei Reductionen unter Abwesenheit von Säuren fast ausschliefslich stattfindet.

Die Erklärung der Isomerie der beiden Desoxybenzoïnpinakone kann keine Schwierigkeiten bieten, da dieselben, wie aus der oben aufgestellten Formel ersichtlich ist, zwei aneinander gebundene asymmetrische Kohlenstoffatome enthalten. Es wären mithin ebenso viel Pinakone dieser Constitution möglich, als es Weinsäuren giebt. Beide Pinakone erwiesen sich bei einer Prüfung ihrer Lösungen in Aceton und in Eisessig in $200 \mathrm{~mm}$ langer Schicht als optisch inactiv. Sonach mufs das eine, vielleicht das niedriger schmelzende $\beta$-Desoxybenzoinpinakon, der Mesoweinsäure entsprechend, durch intramoleculare Compensation optisch inactiv sein, das andere, wahrscheinlich das $\alpha$-Desoxybenzoinpinakon, aber dadurch, dafs es analog der Traubensäure eine Verbindung gleicher Molecule rechts- und linksdrehenden Pinakons vorstellt, wie folgende Formelbilder veranschaulichen : 

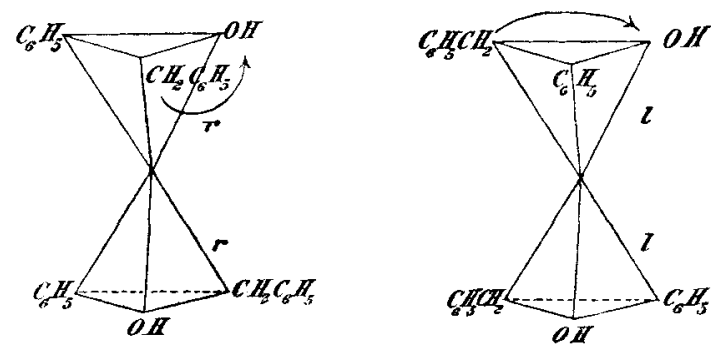

Verbindung von rechts- und linksdrehendem Pinakon.

Durch intramoleculare Compensation optisch inactiv.

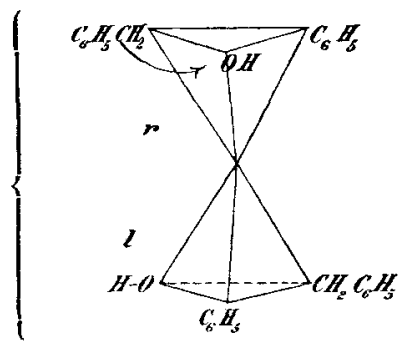

Von einem Versuche der Trennung der beiden Bestandtheile wie bei der Traubensäure in eine rechts- und eine linksdrehende Substanz mufste von vornherein abgesehen werden wegen der Unlöslichkeit der Pinakone in Wasser.

Dem von Jena nnd Limpricht durch Reduction von Benzoïn mit $\mathrm{NaOC}_{2} \mathrm{H}_{5}$ in Alkohol erhaltenen Körper vom Schmelzpunkt $61^{\circ}$ und der Formel $\mathrm{C}_{28} \mathrm{H}_{26} \mathrm{O}_{2}$ kommt jedenfalls die Formel :

$$
\begin{aligned}
& \mathrm{C}_{6} \mathrm{H}_{5} \cdot \mathrm{CH}(\mathrm{OH}) \cdot \mathrm{CH} \cdot \mathrm{C}_{6} \mathrm{H}_{5} \\
& \mathrm{C}_{6} \mathrm{H}_{5} \cdot \mathrm{CH}(\mathrm{OH}) \cdot \stackrel{\mathrm{CH} \cdot \mathrm{C}_{6} \mathrm{H}_{5}}{ }
\end{aligned}
$$


zu, da ja in alkalischer Lösung aus Benzoïn zuerst Hydrobenzoïn gebildet wird, welches durch Entziehung je einer Hydroxylgruppe aus zwei Moleculen in einen Körper obiger Constitution übergehen könnte. Doch ist derselbe nicht als ein Pinakon zu bezeichnen.

\section{Die beiden Dichlorstilbene oder Tolandichloride.}

Unter den Abkömmlingen des Stilbens erregten die beiden Dichlorstilbene berechtigtes Interesse, besonders seit $\mathrm{W}$ islicenus die van t'Hoff und Le Bel'sche Theorie über die räumliche Lagerung der Atome in organischen Verbindungen erweiterte.

Fast gleichzeitig wurden die Tolandichloride beobachtet von $\mathrm{Zin}$ in*), welcher sie bei der Einwirkung von Zink auf eine alkoholische Tolantetrachloridlösung erhielt :

$$
\frac{\mathrm{C}_{6} \mathrm{H}_{5}-\mathrm{CCl}_{2}}{\text { Tolantetrachlorid }}+\mathrm{Zn}=\mathrm{ZnCl}_{2}+\underbrace{\frac{\mathrm{C}_{6} \mathrm{H}_{5}-\mathrm{CCl}_{5}-\mathrm{C}-\mathrm{Cl}}{\mathrm{C}_{6} \mathrm{H}_{5}-\mathrm{C}-\mathrm{Cl}}}_{\text {Tolandichlorid }},
$$

und von $L$ i mpricht und Schwanert**), die beim Erhitzen von Stilben und Phosphorpentachlorid das Auftreten derselben

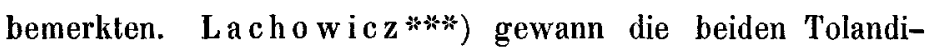
chloride aus dem Tolantetrachlorid, indem er Wasserstoff, entwickelt aus Eisessig und Eisen, als chlorentziehendes Mittel anwendete.

Die beiden Tolandichloride unterscheiden sich vollkommen in ihren physikalischen Eigenschaften. Während das eine, bislang als $\beta$-Tolandichlorid bezeichnete, in Nadeln vom Schmelzpunkte $63^{\circ}$ krystallisirt, bildet das andere, das $\alpha$-Tolandichlorid, bei $143^{\circ}$ schmelzende rhombische Tafeln. 1 Gewichtstheil der ersteren Verbindung bedarf zur Lösung bei gewöhn-

*) Ber. d. deutsch. chem. Ges. 4, 289.

**) Daselbst 4, 379.

***) Daselbst 1 ₹, 1164 . 
licher Temperatur nur 7,8 Theile Weingeist, während die letztere sich erst in dem 150 fachen Gewichte absoluten Alkohols zu lösen vermag. Zur Erklärung der Verschiedenheit der beiden Tolandichloride hat J. Wislicenus*) geometrische Gründe angeführt und an der Hand von Thatsachen entwickelt, dafs das hochschmelzende Dichlorid das plansymmetrische :

$$
\mathrm{C}_{6} \mathrm{H}_{5}-\mathrm{C}-\mathrm{Cl}
$$

das niederschmelzende das axialsymmetrische :

$$
\mathrm{C}_{6} \mathrm{H}_{5}-\mathrm{C}-\mathrm{Cl}
$$

vorstelle. Doch war ein Verhältnifs der Polymerie der beiden Verbindungen zueinander a priori nicht ausgeschlossen.

Da die Siedepunkte einander polymerer Substanzen immer weit auseinanderliegen, so hätte, falls das Molecul des höher schmelzenden Körpers ein Vielfaches desjenigen des niederschmelzenden gewesen wäre, der Siedepunkt des ersteren im Vergleich zu dem des andern viel höher gefunden werden müssen. Eine Bestimmung der Siedepunkte bei gewöhnlichem Luftdrucke konnte nicht zum Ziele führen, weil die Tolandichloride, wie schon Limpricht und Schwanert ${ }^{* *}$ ) fanden, beim Destilliren in einander umgewandelt werden. Ich kann diese Angabe nur bestätigen. Es ist gleichgültig, ob man das bei $63^{\circ}$ oder das bei $143^{\circ}$ schmelzende Chlorid der Destillation unterwirft, immer geht bei $320^{\circ}$ ein Gemisch beider über, und wenn man das Destillat untersucht, findet man, dafs auf 7 Theile des niederschmelzenden nur 3 Theile des hochschmelzenden kommen. Es ist dies begreiflich, wenn

*) Joh. Wislicenus : Ueber die räumliche Anordnung der Atome in organ. Molec. 1887. Seite 23.

**) Ber. d. deutsch. chem. Ges. 4, 379. 
man die von Wislicenus*) gegebenen Erklärungen über die Umwandlung ungesättigter Verbindungen in geometrisch Isomere durch Wärme auf den vorliegenden Fall anwendet.

Sobald infolge der Wärmewirkung die Bindungsenergieen der Atome im Molecul gelockert werden, vermag das eine Tolandichlorid in das andere überzugehen. Natürlicherweise treten alsdann die der begünstigteren, also der axialsymmetrischen Form entsprechenden Molecule in gröfserer Zahl auf als die der plansymmetrischen Configuration, und zwar wird das relative Verhältnifs beider sich mit der Temperatur ändern, für einen bestimmten Wärmegrad aber ein constantes sein.

Bei stark vermindertem Luftdrucke destilliren die beiden Tolandichloride vollkommen unverändert und zwar siedet das Chlorid vom Schmelzpunkt $63^{\circ}$ bei einem Druck von $18 \mathrm{~mm}$ bei $178^{\circ}$, das Chlorid vom Schmelzpunkt $143^{\circ}$ unter demselben Drucke bei $183^{\circ}$. Die Siedepunkte liegen also so nahe, dafs an eine Erklärung der Verschiedenheit der beiden Verbindungen durch die Annahme, die eine sei der andern polymer, nicht zu denken war. Dieses Resultat wurde bestätigt durch eine Bestimmung der Dampfdichte nach Vict. Meyer bei $360^{\circ}$. Die erhaltenen Zahlen sind folgende :

I. $0,11 \mathrm{~g}$ Tolandichlorid (Schmelzpunkt $143^{\circ}$ ) lieferten $10,85 \mathrm{cbcm}$ verdrängte Luft, gemessen bei einer Temperatur von $20^{\circ}$ und $732 \mathrm{~mm}$ Barometerstand.

II. $0,1400 \mathrm{~g}$ Tolandichlorid (vom Schmelzpunkt $63^{\circ}$ ) gaben bei $24,5^{\circ}$ und $752 \mathrm{~mm}$ Barometerstand $15,0 \mathrm{cbcm}$ verdrängte Luft.

Berechnete Dampfdichte für $\mathrm{C}_{14} \mathrm{H}_{10} \mathrm{Cl}_{2}$ 8,61 Gefundene Dampfdichte

$\begin{array}{cc}\text { I. } & \text { II. } \\ 8,74 & 8,23 .\end{array}$

Ferner ergab eine Moleculargewichtsbestimmung nach der Ra oult'schen Gefriermethode, dafs beiden Dichloriden dieselbe Molecularformel $\mathrm{C}_{14} \mathrm{H}_{10} \mathrm{Cl}_{2}$ zukommt :

*) J. Wislicenus : Ueb. d. räuml. Anordn. d. Atome. Leipzig, 1887, S. 54. 


\begin{tabular}{|c|c|c|c|c|c|c|}
\hline \multicolumn{2}{|c|}{ Angewendet : } & \multicolumn{2}{|c|}{ Gefrierpunkt } & \multirow{2}{*}{ 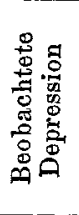 } & \multirow{2}{*}{ 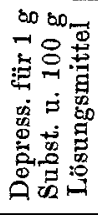 } & \multirow{2}{*}{ 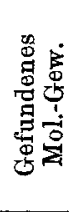 } \\
\hline 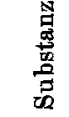 & 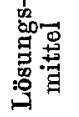 & 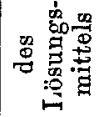 & 量 & & & \\
\hline
\end{tabular}

I. Bestimmungen in Eisessig :

a) Tolandichlorid rom Schmelzpunkt $63^{\circ}$. Mol.-Gew. 248,8.

$$
|0,315 \mathrm{~g}| 18,533 \mathrm{~g}\left|15,68^{\circ}\right| 15,40^{\circ}\left|0,28^{\circ}\right| 0,1648^{\circ} \mid 236,7
$$

b) Tolandichlorid vom Schmelzpunkt $143^{\circ}$. Mol.-Gew. 248,8.

$$
\mid 0,091 \text { g } \mid 21,65 \text { g }\left|15,67^{\circ}\right| 15,6^{\circ}\left|0,07^{\circ}\right| 0,1666^{\circ} \mid 234
$$

II. Bestimmungen in Benzol :

a) Tolandichlorid rom Schmelzpunkt $63^{\circ}$. Mol.-Gew. 248,8.

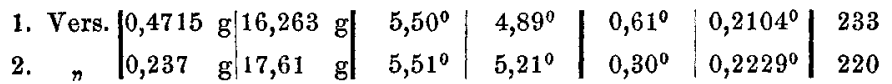

b) Tolandichlorid rom Schmelzpunkt $143^{\circ}$. Mol.-Gew. 248,8.

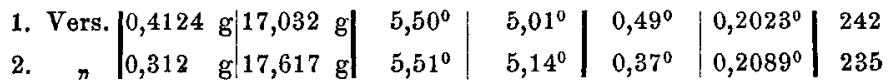

Aus all den angeführten Thatsachen und Versuchen geht hervor, dafs die beiden Tolandichloride wirklich structuridentisch sind und dafs ihre Verschiedenheit nur durch $\mathrm{Zu}-$ hülfenahme räumlicher Vorstellungen über die Anordnung ihrer Elementaratome erklärt werden kann. Die gleichzeitige Bildung der Dichlorstilbene aus dem Tolantetrachlorid konnte ebenfalls nur in geometrischen Verhältnissen ihren Grund haben.

Wislicenus führte aus, dafs von den für das Tolantetrachlorid möglichen, einander nicht congruenten drei Configurationen :

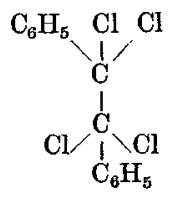

I.

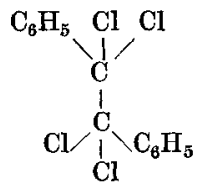

II.<smiles>CCCCC(Cl)(Cl)C(Cl)(Cl)Cl</smiles>

III.

die I und II entsprechenden Molecule das axialsymmetrische Tolandichlorid : 


$$
\stackrel{\mathrm{C}_{6} \mathrm{H}_{5}-\mathrm{C}-\mathrm{Cl}}{\mathrm{Cl}-\mathrm{C}-\mathrm{C}_{6} \mathrm{H}_{5}},
$$

die nach III zusammengesetzten das plansymmetrische :<smiles>ClC(Cl)=C(Cl)c1ccccc1</smiles>

liefern, wenn die correspondirenden Chloratome herausgenommen werden. Bei sehr niederen Temperaturen wird vielleicht das Tolantetrachlorid nur aus kleinsten Theilchen bestehen, die den Formeln I und II entsprechen, in welchen den wirksamsten Affinitäten am meisten Rechnung getragen ist. Mit steigender Temperatur tritt jedoch ein Vorgang ein, welcher wohl mit der Dissociation zu vergleichen ist. In einem Theil der Molecule überwinden energischere Wärmestöfse die Anziehungskräfte der correspondirenden Chlorund Phenylgruppen, und es findet eine Rotation der beiden Kohlenstoffsysteme um einander statt. Letztere nehmen nach einander die in den Figuren I, II und III auf der vorhergehenden Seite gezeichneten Stellungen ein. Die in diesem Zustande befindlichen Molecule werden mit weiter steigender Temperatur vermehrt und damit auch die Anzahl der, der weniger begünstigten Configuration (III) entsprechenden.

Dieses Wachsen hat jedoch eine Grenze, welche erreicht ist, wenn alle Molecule derartige Rotationen ausführen; dann enthält aber das Tolantetrachlorid ungefähr auf 2 Molecule der begünstigteren eines der weniger begünstigten Configuration. Diese Verhältnisse wurden einer Untersuchung unterworfen, deren Resultate nachstehend angeführt werden.

\section{Darstellung des Tolantetrachlorids.}

Hanhart*) und Onafrowicz**) hatten beim Erhitzen von Benzotrichlorid mit Kupferpulver für sich oder in Benzol-

*) Ber. d. deutsch. chem. Ges. 15, 901.

*) Daselbst 17,833 . 
lösung die Bildung von Tolantetrachlorid beobachtet. Behufs Darstellung desselben nach dieser Methode verwendete ich den zwischen 211 und $220^{\circ}$ siedenden Antheil des käuflichen Benzotrichlorids. Aus $1 \mathrm{~kg}$ des letzteren erhielt ich $600 \mathrm{~g}$ dieser Fraction. Hiervon wurden $200 \mathrm{~g}$ mit der gleichen Menge Benzol verdünnt und mit $80 \mathrm{~g}$ Kupferpulver vier Stunden lang am Rückflufskühler erhitzt. Nach dem Abfiltriren und Auswaschen des zurückbleibenden Kupfers und Kupferchlorürs mit Benzol destillirte ich dasselbe aus dem braun gefärbten Filtrate $a b$, trennte die nach längerem Stehen sich ausscheidenden Krystalle von der dunklen Mutterlauge und krystallisirte sie so lange um, bis sie den richtigen Schmelzpunkt von $162^{\circ}$ zeigten. $200 \mathrm{~g}$ Benzotrichlorid (Siedepunkt 211 bis $220^{\circ}$ ) lieferten 50 bis $60 \mathrm{~g}$ des fast reinen Tolantetrachlorides.

Das in anderer Weise aus Benzil oder Chlorobenzil und Phosphorpentachlorid durch 6 bis 7 stündiges Erhitzen auf $200^{\circ}$ in zugeschmolzenen Röhren erhaltene Tetrachlorid zeichnet sich durch grofse Reinheit aus; doch ist die Ausbeute auch hier keine quantitative, indem immer ein Theil Chlorobenzil sich der Einwirkung des Phosphorpentachlorides entzieht.

Behufs Gewinnung gröfserer Mengen Tolantetrachlorids ist die erstere Methode der zweiten vorzuziehen.

Einwirkung von Zink auf Tolantetrachlorid.

Um nachzuweisen, dafs mit steigender Temperatur die Anzahl der weniger begünstigten Configurationen des Tolantetrachlorids zunimmt, wurden die bei Einwirkung von Zink auf dasselbe bei verschiedenen Temperaturen entstehenden Mengen der beiden Tolandichloride genau bestimmt.

Der Procefs wurde ausgeführt :

1) Bei einer Temperatur von $20^{\circ}$ in äthylalkoholischer Lösung. 
2) Bei einer Temperatur von $80^{\circ}$ in siedender äthylalkoholischer Lösung.

3) Bei einer Temperatur von $130^{\circ}$ in siedender amylalkoholischer Lösung. Bei diesen Temperaturen verläuft die Reaction vollkommen quantitativ, indem sich alles Tolantetrachlorid nur in die beiden Tolandichloride umwandelt. Es wurde versucht, den Procefs auch bei noch höheren Temperaturen auszuführen, und zu dem Ende Tolantetrachlorid mit Kupfer auf $180^{\circ}$ erhitzt. Dabei trat jedoch Salzsäure auf und es entstand aufser den Tolandichloriden noch eine schwarzbraune, nicht krystallisirende Substanz, so dafs von der Anwendung höherer Temperaturen abgesehen wurde.

Die Einwirkung des Zinks auf das Tolantetrachlorid und die Trennung der entstandenen Producte wurde in folgender Weise ausgeführt.

1) Versuch bei $20^{\circ}$.

$20 \mathrm{~g}$ feingepulvertes Tolantetrachlorid wurden mit $8 \mathrm{~g}$ eines gereinigten Zinkstaubes und $200 \mathrm{~g}$ Aethylalkohol bei gewöhnlicher Temperatur in einer gut verstopften Kochflasche stehen gelassen. Von Zeit zu Zeit wurde umgeschüttelt. Nach Verlauf von 14 Tagen zeigte sich, dafs das feinkörnige Tetrachlorid ganz verschwunden und an seine Stelle eine geringe Menge von in Blättchen krystallisirendem Dichlorid getreten war. Nach dem Erwärmen auf dem Wasserbade, zum Zwecke der Lösung des ausgeschiedenen Chlorides, filtrirte ich vom überschüssigen Zinkstaube ab, wusch mit Alkohol gut aus und destillirte denselben aus dem Filtrate möglichst ab. Um das Chlorzink zu entfernen, schüttelte ich das zurückbleibende Oel mit Wasser durch, wobei es zu einer Krystallmasse erstarrte. Diese sammelte ich auf einem Filter, wusch gut aus und löste sie nach dem Trocknen wieder in etwa $150 \mathrm{cbcm}$ heifsen absoluten Alkohols auf. Beim Erkalten 
schied sich die Hauptmenge des entstandenen plansymmetrischen Tolandichlorides aus. Nach dem Abfiltriren und Auswaschen desselben wurde aus dem Filtrate der Alkohol gröfstentheils verdampft und die hinterbleibende ölige Flüssigkeit durch Abkühlen und Umschütteln zum schnellen Krystallisiren gebracht. Das axialsymmetrische Tolandichlorid scheidet sich so in kleinen Nädelchen ab. Der Krystallbrei wurde ungefähr 24 Stunden stehen gelassen, alsdann mit etwa $150 \mathrm{cbcm}$ absoluten Alkohols behufs Lösung des axialsymmetrischen Chlorides geschüttelt, der ungelöste Rückstand schnell auf ein Filter gebracht und so lange mit Alkohol ausgewaschen, bis er bei $143^{\circ}$ schmolz. Diese Operation wurde nochmals wiederholt. Das Filtrat engte ich auf etwa $50 \mathrm{cbcm}$ ein. Beim Erkalten krystallisirten Nadeln des axialsymmetrischen Dichlorides aus, die auf einem Filter gesammelt wurden. Die durchgelaufene Lösung wurde durch Erhitzen auf dem Wasserbade stark concentrirt, durch Kühlen und Schütteln zum Krystallisiren gebracht nach 24 Stunden mit etwa 80 bis $100 \mathrm{cbem}$ Alkohol aufgenommen, der verbleibende sehr geringe Rückstand auf einem Filter gesammelt und ausgewaschen. Dies wurde so lange wiederholt, bis kein plansymmetrisches Chlorid mehr vorhanden war. Aus der verbleibenden Lösung von axialsymmetrischem Chlorid verdampft man den Alkohol, wäscht zur Entfernung noch vorhandener sehr geringen Mengen von Zinkchlorid mit Wasser, läfst das feuchte Tolandichlorid an der Luft trocknen, zieht mit Aether aus, verdunstet letzteren und vereinigt mit dem Rückstande die vorher ausgeschiedenen Nadeln. Selbstverständlich wurden die zum Sammeln der Niederschläge dienenden Filter nach Entfernung der Rückstände mit Alkohol extrahirt und die erhaltene Lösung zum jeweiligen Filtrate gegeben. Die durch längeres Stehen im Exsiccator völlig getrockneten Tolandichloride wurden alsdann gewogen, in Benzol gelöst und ein 
eventueller Rückstand vom Gewichte derselben in Abzug gebracht.

\section{2) Versuch bei $80^{\circ}$.}

In einem geräumigen Gefäfse wurden $20 \mathrm{~g}$ Tolantetrachlorid mit $200 \mathrm{~g}$ Alkohol zum Sieden erhitzt.

Ich nahm hierauf das Gefäfs vom Wasserbade weg und trug, gleichviel ob sich alles Tolantetrachlorid gelöst hatte oder nicht, $8 \mathrm{~g}$ Zinkstaub nach und nach etwa im Verlaufe von 5 Minuten ein. Würde man den Zinkstaub auf einmal hinzufügen, so könnten durch die sehr heftige Reaction Verluste stattfinden. Um die Umsetzung zu vollenden wurde das Ganze noch 20 bis 25 Minuten am Rückflufskühler erhitzt, abfiltrirt, und bezüglich der Trennung der beiden Tolandichloride genau in der oben beschriebenen Weise verfahren.

\section{3) Versuch bei $130^{\circ}$.}

Es wurden $20 \mathrm{~g}$ Tolantetrachlorid in $200 \mathrm{~g}$ Amylalkohol gelöst, die Lösung bis zum Kochen erhitzt, $8 \mathrm{~g}$ Zinkstaub allmählich eingetragen, noch 20 Minuten lang gekocht, in einen Fractionskolben filtrirt, mit heifsem Amylalkohol gut nachgewaschen und derselbe gröfstentheils abdestillirt, so zwar, dafs ein in die Flüssigkeit eingesenktes Thermometer nicht über $145^{0}$ stieg. Da beide Tolandichloride in geringer Menge mit den Amylalkoholdämpfen flüchtig sind, so wurde der abdestillirte Amylalkohol nochmals fractionirt und die letzten Theile Flüssigkeit, die im Siedekölbchen blieben, mit der Hauptmasse der Tolandichloride vereinigt. Die Trennung wurde in der obigen Art und Weise ausgeführt. Die erhaltenen Zahlen sind folgende :

$20 \mathrm{~g}$ Tolantetrachlorid $=15,56$ Dichloride. 


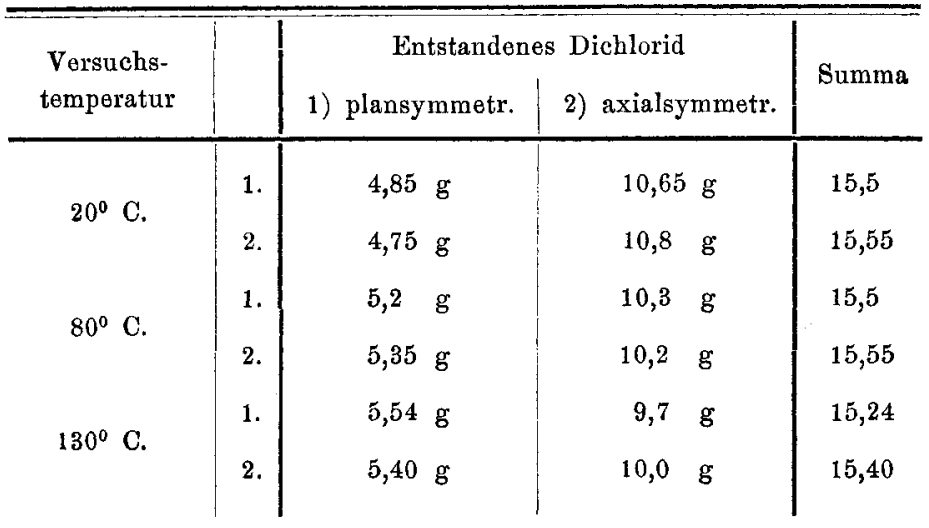

Um diese Werthe, wenigstens die für $80^{\circ}$ und $130^{\circ}$ erhaltenen, zu controlliren, wurde in 2 weiteren Versuchsreihen auf die Gewinnung des axialsymmetrischen Chlorides verzichtet und nur das plansymmetrische auf folgende Weise bestimmt.

Die die Reactionsproducte enthaltende, vom Zinkstaub abfiltrirte Lösung wurde eingeengt, noch heifs in einen Mefskolben von $200 \mathrm{cbcm}$ Inhalt gegeben, mit absoluten Aethylbeziehungsweise Amyl-Alkohol nachgespült und damit bis zur Marke aufgefüllt und umgeschüttelt. Nach 24 stündigem Stehen an einem Orte gleichmäfsiger, mittlerer Temperatur wurde das ausgeschiedene plansymmetrische Chlorid auf einem Saugfilter gesammelt und mit einer bei derselben Temperatur gesättigten Lösung des Chlorides in Alkohol sorgfältig ausgewaschen. Die Rückstände wurden getrocknet und gewogen, schliefslich in Benzol gelöst und zurückbleibendes Zinkoxychlorid von dem Gewichte in Abzug gebracht. Um die in den $200 \mathrm{cbcm}$ gelöste Menge des plansymmetrischen Tolandichlorides zu erfahren, wurden daneben in zwei Kölbchen von je $200 \mathrm{cbcm}$ Inhalt abgewogene Mengen dieses Chlorides in warmem Aethyl- und Amyl-Alkohol gelöst und bis zur Marke Alkohol nachgefüllt. Die nach 24 Stunden wieder ausgeschiedene Menge des Chlorides filtrirte ich ab und wog sie 
nach dem Trockenen. Wegen der geringen Löslichkeit des plansymmetrischen Chlorides in Alkohol kann von dem Fehler, welcher durch Eintrocknen der den Krystallen anhaftenden concentrirten Lösung entsteht, ganz abgesehen werden. Derselbe beträgt höchstens $0,01 \mathrm{~g}$. Die erhaltenen Zahlen sind folgende :

Angewendet $20 \mathrm{~g}$ Tolantetrachlorid.

I. bei $80^{\circ}$ C. : 1) ausgeschiedenes plansym. Chlorid $4,2 \mathrm{~g}$; in $200 \mathrm{cbcm}$ gelöst

Summa $\frac{1,08 \pi}{5,28 \mathrm{~g} .}$

2) ausgeschiedenes plansym. Chlorid $4,31 \mathrm{~g}$;

in $200 \mathrm{cbcm}$ gelöst

zusammen $\frac{1,08 \mathrm{n}}{5,39 \mathrm{~g} .}$

II. bei $130^{\circ}$ O. : 1) ausgesehiedenes plansym. Chlorid $4,55 \mathrm{~g}$; in $200 \mathrm{cbcm}$ gelöst

zusammen $\frac{1,01 \mathrm{n}}{5,56 \mathrm{~g} .}$

2) ausgeschiedenes plansym. Chlorid

$4,64 \mathrm{~g}$;

in $200 \mathrm{cbcm}$ gelöst

Summa $\frac{1,01 \pi}{5,65 \mathrm{~g} .}$

Wie ersichtlich bekam ich hierbei etwas höhere Werthe als früher; doch ist dies ganz erklärlich, da nach ersterer Methode die Trennung der beiden Dichloride keine vollkommene sein kann.

Durch diese Versuche ist mit Sicherheit nachgewiesen, dafs mit steigender Temperatur die Menge des plansymmetrischen Tolandichlorides zunimmt.

Betrachtet man jedoch die für dasselbe erhaltenen Zahlen näher, so ergiebt sich dafs sie der Theorie nach zu hoch ausgefallen sind. Da, wie früher entwickelt wurde, im Tolantetrachlorid auf 2 Molecule der begünstigten im Maximum nur eines der weniger begünstigten Configuration kommen kann, und $20 \mathrm{~g}$ Tetrachlorid 15,56 $\mathrm{g}$ Dichlorid entsprechen, so dürften sich im vorliegenden Falle höchstens $\frac{15,56}{3}=5,19 \mathrm{~g}$ plansymmetrisches Tolandichlorid bilden, welche Grenze aber 
bei $80^{\circ}$ und $130^{\circ}$ überschritten wurde. Diese Unregelmäfsigkeit klärte sich jedoch völlig auf, als es gelang, ein Zwischenproduct aufzufangen.

Wurden nämlich die oben angegebenen Bedingungen in Bezug auf die Entchlorung des Tetrachlorides nicht genau eingehalten, wendete man also zu wenig Zinkstaub an, oder verdünnte man die Lösungen mehr und erhitzte kürzere Zeit, so erhielt man nie übereinstimmende Zahlen für die beiden Tolandichloride aus verschiedenen Portionen. In der Regel fielen die für das plansymmetrische Chlorid alsdann zu hoch aus. In solchen Fällen konnten jedoch mit Sicherheit immer im abgeschiedenen plansymmetrischen Dichlorid kleine Mengen einer nicht in Blättchen krystallisirenden, körnigen, farblosen, bei $149^{\circ}$ schmelzenden Substanz nachgewiesen werden, welche wegen ihres Schmelzpunktes und ihrer anderen Krystallform nicht bei der Reaction unverändert gebliebenes Tolantetrachlorid sein konnten.

Dieselbe enthielt Chlor und gab bei der Analyse folgende Werthe :

I. $0,2407 \mathrm{~g}$ Substanz lieferten $0,5205 \mathrm{CO}_{2}$ und $0,0775 \mathrm{H}_{2} \mathrm{O}$;

II. $0,2204 n n \pi \quad 0,4767 n \pi 0,063 n$

III. $0,2232 n n \quad n \quad 0,4830 n n 0,0738$ n

IV. 0,4560 " $" n$ nach dem Glühen mit $\mathrm{CaO}$, Aufnehmen in Salpetersäure und Fallen mit Silbernitrat : $0,7000 \mathrm{Ag}_{2} \mathrm{Cl}_{2} ;$

V. 0,394 g Substanz glühte ich mit Kalk, Iöste alles in Salpetersäuro auf und verdünnte die Lösung auf $500 \mathrm{cbcm}$. $200 \mathrm{cbcm}$ davon brauchten beim Titriren $16,7 \mathrm{cbcm}$ oiner Zehntelsnormalsilberlösung.

\begin{tabular}{|c|c|c|c|c|c|c|}
\hline & \multirow{2}{*}{$\begin{array}{l}\text { Berechnet für } \\
\quad \mathrm{C}_{88} \mathrm{H}_{20} \mathrm{Cl}_{6}\end{array}$} & \multicolumn{5}{|c|}{ Gefunden } \\
\hline & & I. & II. & III. & IV. & $\overline{\mathrm{V}}$. \\
\hline $\mathrm{C}$ & 59,11 & 58,98 & 58,99 & 59,02 & - & - \\
\hline $\mathbf{H}$ & 3,52 & 3,58 & 3,18 & 3,67 & - & - \\
\hline $\mathrm{Cl}$ & 37,37 & - & - & - & 37,98 & 37,51 \\
\hline
\end{tabular}


Die erhaltenen Zahlen entsprechen der Zusammensetzung eines Ditolanhexachlorids, dessen Entstehung durch folgende Gleichung versinnlicht wird :

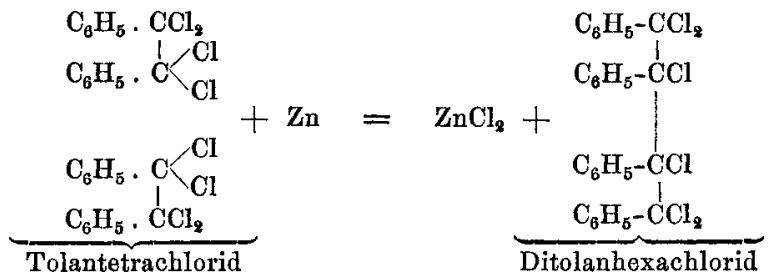

Die Substanz krystallisirt anscheinend im rhombischen System, ist in Alkohol und Aether schwer, in Benzol dagegen leicht löslich. Aus letzterem scheidet sie sich gewöhnlich in Warzen ab. Ihr Schmelzpunkt liegt bei $150^{\circ}$. Sie ist nicht unzersetzt flüchtig. Unter den Destillationsproducten treten vor allem die beiden Tolandichloride auf, aufserdem entweicht Salzsäure.

Die Bildung des Ditolanhexachlorides aus Tolantetrachlorid erfolgt nicht quantitativ. Läfst man auf das letztere so viel Zinkstaub wirken, dafs es gerade zersetzt ist, so sind aus $20 \mathrm{~g}$ Tetrachlorid etwa $9,3 \mathrm{~g}$ des Ditolanhexachlorids neben $7,4 \mathrm{~g}$ des axialsymmetrischen Tolandichlorides entstanden, d. i. ungefähr auf 1 Molecul Hexachlorid 2 Molecule Dichlorid. Plansymmetrisches Chlorid hat sich dabei überhaupt nicht gebildet. Erst bei der weiteren Einwirkung von Zinkstaub auf das Hexachlorid entsteht dasselbe, und zwar lieferten $10 \mathrm{~g}$ Hexachlorid :

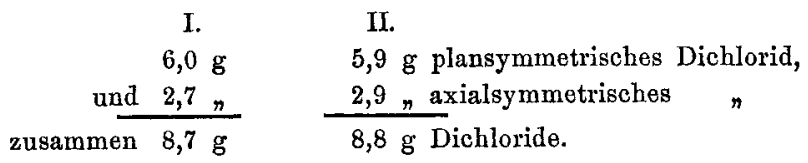

Dieses Verhältnifs der beiden Dichloride kann nicht auffallen, wenn man die räumliche Formel des Hexachlorides näher ins Auge fafst. Da es zwei asymmetrische Kohlenstoffatome enthält, und, wie ein Versuch zeigte, seine Lösung 
den polarisirten Lichtstrahl nicht aus seiner Schwingungsebene ablenkt, es also wahrscheinlich durch intramoleculare Compensation optisch inactiv ist, so kommt ihm jedenfalls folgende Configuration $\mathrm{zu}$ :

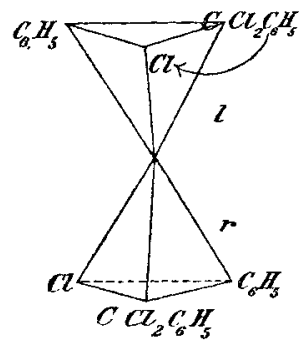

Wenn man dieser Verbindung weiter 2 Chloratome unter Bildung von Tolandichloriden entzieht, so müssen immer diejenigen Chloratome aus den $\mathrm{CCl}_{2} \mathrm{C}_{6} \mathrm{H}_{5}$-Gruppen herausgenommen werden, welche sich bei den Drehungen der verschiedenen Kohlenstoffsysteme jedesmal am nächsten stehen.

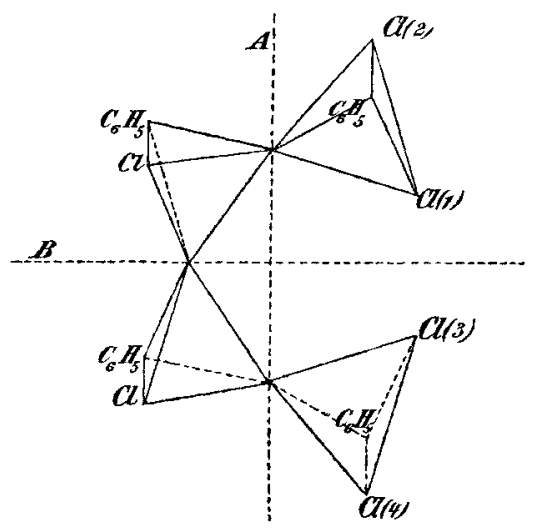

Diese Verhältnisse sind von zu complicirter Art, als dafs sich jetzt schon übersehen liefse, welche $\mathrm{Cl}$-Atome austreten. Doch vermag man sich über die aus dem Hexachlorid entstehenden Mengen der Dichloride zu orientiren. Denkt man 
sich in obiger Figur die mit (1) und (3) bezeichneten Chloratome beseitigt, so wird vielleicht vorübergehend ein Körper entstehen, in welchem die 4 Kernkohlenstoffatome unter einander verbunden sind, der aber sofort in die beiden Tolandichloride zerfällt. Diese Spaltung kann ebensowohl in der Richtung der Linie A, als in der der Linie B geschehen. Der Erfolg wird hier in beiden Fällen der gleiche sein. Es entsteht immer auf 1 Molecul des axialsymmetrischen 1 Molecul des plansymmetrischen Chlorides. Wenn das Zink die Chloratome (2) und (4) oder (1) und (4) bindet, müssen ebenfalls die beiden Tolandichloride in gleich grofser Menge auftreten. Nimmt man aber die mit (2) und (3) bezeichneten Chloratome heraus,

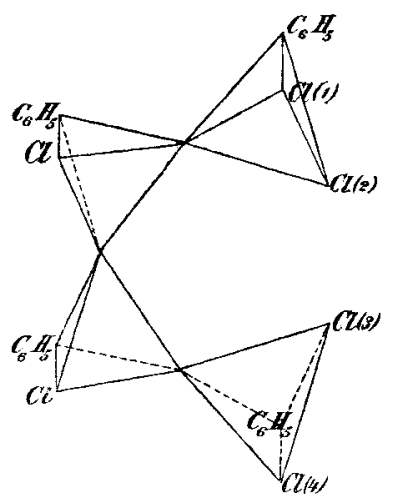

so kann sich nur plansymmetrisches Tolandichlorid bilden. Letzteres mufs mithin aus dem Ditolanhexachlorid bei der weiteren Chlorentziehung in gröfserer Menge entstehen als das axialsymmetrische; dies ist in der That, wie die oben mitgetheilten Versuche zeigen, der Fall.

Das Ditolanhexachlorid läfst sich auch gewinnen, indem man Tolantetrachlorid mit den Dichloriden in Reaction bringt. Uebergiefst man ein Gemisch gleicher Molecule von Tolantetrachlorid und plansymmetrischem Dichlorid mit etwa 
der 30 fachen Menge Alkohol und erhitzt so lange bis sich alles gelöst hat, so krystallisirt beim Erkalten reines Ditolanhexachlorid heraus, mit dem Schmelzpunkt, den Eigenschaften und dem Chlorgehalte desselben.

$0,2695 \mathrm{~g}$ Substanz gaben $0,4111 \mathrm{Ag}_{2} \mathrm{Cl}_{\mathbf{2}}$.

$\begin{array}{ccc} & \text { Berechnet für } & \text { Gefunden } \\ \text { Cl } & \mathrm{C}_{28} \mathrm{H}_{20} \mathrm{Cl}_{6} & \\ & 37,37 & 37,73 .\end{array}$

Centrisch-symmetrisches Tolandichlorid in der gleichen Weise mit Tetrachlorid erhitzt, liefert kein Hexachlorid. Aus der Lösung lassen sich beide unverändert wieder erhalten. Erhitzt man sie dagegen $1 / 2$ Stunde lang im Oelbade auf $180^{\circ}$, also auf eine Temperatur, bei welcher die Tolandichloride sich noch nicht in einander zu verwandeln beginnen, so findet die Umsetzung in Hexachlorid statt, wenn auch nicht vollständig. Durch Umkrystallisiren des erkalteten Rückstandes aus Alkohol und Benzol erhält man erst unverändertes Tolantetrachlorid, dann aber die bei $150^{\circ}$ schmelzenden Krystalle des Ditolanhexachlorides.

Die Verschiedenheit des Verhaltens der beiden Dichloride gegenüber dem Tetrachlorid erklärt sich beim Betrachten der räumlichen Formeln derselben. Im axialsymmetrischen Chlorid mufs die Intensität der doppelten Bindung der beiden Kohlenstoffatome, weil dieselbe durch die gröfseren Affinitäten zwischen Chlor und Phenyl unterstützt wird, auch eine gröfsere sein wie im plansymmetrischen. Infolge dessen wird das letztere unter gewissen Einflüssen die eine seiner Bindungen leichter und bei niedrigerer Temperatur lösen als das centrischsymmetrische.

Beim Einleiten von Chlorgas in die geschmolzenen Dichloride entstehen neben öligen Flüssigkeiten gewisse Mengen Ditolanhexachlorides, welches nach dem Umkrystallisiren constant bei $150^{\circ}$ schmilzt. Dasselbe wird bei der weiteren 
Behandlung mit Chlor nur sehr schwierig angegriffen, doch geht es zum Theil in Tolantetrachlorid über.

Merkwürdigerweise zeigt das Ditolanhexachlorid nach der $\mathrm{R}$ a 0 u $1 \mathrm{t}$ 'schen Methode nur das halbe Moleculargewicht. Die erhaltenen Zahlen sind die folgenden :

\begin{tabular}{|c|c|c|c|c|c|c|c|}
\hline & \multicolumn{2}{|c|}{ Angewendet } & \multicolumn{2}{|c|}{ Gefrierpunkt } & \multicolumn{2}{|c|}{ 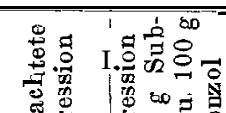 } & \multirow{2}{*}{ 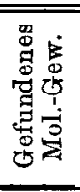 } \\
\hline & Substanz & Benzol & $\begin{array}{c}\text { des } \\
\text { Benzols }\end{array}$ & $\begin{array}{c}\text { der } \\
\text { Lösung }\end{array}$ & 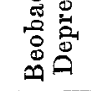 & 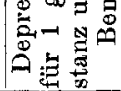 & \\
\hline 1. & $0,1556 \mathrm{~g}$ & $15,1 \mathrm{~g}$ & $5,51^{\circ}$ & $5,32^{0}$ & $0,19^{\circ}$ & $0,184^{\circ}$ & 266 \\
\hline 2. & $0,245 \mathrm{~g}$ & $15,1 \mathrm{~g}$ & $5,51^{\circ}$ & $5,21^{\circ}$ & $0,3^{0}$ & $0,185_{0}$ & 265 \\
\hline
\end{tabular}

Dieses Resultat würde, da es kein Tolantrichlorid gcben kann, darauf hindeuten dafs das Ditolanhexachlorid keine einheitliche Verhindung, sondern ein zusammenkrystallisirendes Gemenge von Tolandi- und -tetrachlorid vorstelle. Gegen diese Annahme sprechen jedoch verschiedene der angefiihrten chemischen Thatsachen, vor allem die Entstehung dieses Körpers aus den 6eiden Dichloriden durch Chlorgas oder durch Umsetzung mit Tolantetrachlorid. Da diese Facta gewichtiger sind als das Resultat der $\mathrm{R}$ a o ul t 'schen Methode, welch' letztere sich noch nicht in ihrer Anwendung auf alle Korperclassen bewährt hat, so mufs die untersuchte Suhstanz als einheitlich, als Ditolanhexachlorid bezeichnet werden.

Dieselbe entsteht bei der Reaction der Chlorentziehung aus dem Tolantetrachlorid auf zweierlei Weise :

1) durch Wiedervereinigung von schon gebildetem plansymmetrischen Chlorid mit Tolantetrachlorid, und

2) durch directe Chlorentziehung.

Vielleicht konnte diese letztere Entstehung bei Anwendung anderer chlorentziehenden Mittel umgangen werden, da dem 
Zink ja im hohen Grade die Eigenschaft, condensirend zu wirken, zukommt. In der That fand $\mathrm{Lachowicz}$ ) bei Anwendung von nascentem Wasserstoff, aus Eisen und Essigsäure entwickelt, auf 1 Theil des plansymmetrischen 5 Theile des axialsymmetrischen Chlorides.

Als ich den Versuch wiederholte, bekam ich zwar nicht dasselbe Resultat, doch immerhin für das plansymmetrische Chlorid niedrigere Zahlen als bei Anwendung von Zinkstaub.

Es wurden erhalten aus $20 \mathrm{~g}$ Tolantetrachlorid :

I. II.

$\left.\begin{array}{lcrr}\text { 1. plansymmetrisches Chlorid } & 4,40 & 4,5 \\ \text { 2. axialsymmetrisches } & n & 11,05 & 10,8\end{array}\right\}$ bei $110^{\circ}$

oder auf 1 Theil des ersteren 2,46 Theile des letzteren.

Sonach sind die für das plansymmetrische Tolandichlorid bei Verwendung von Zink $\mathrm{zu}$ hoch gefundenen Zahlen nur der Bildung eines in beschränkter Menge auftretenden Zwischenproductes zuzuschreiben, welches bei der weiteren Chlorentziehung mehr plansymmetrisches Dichlorstilben geben mufs als axialsymmetrisches.

Im Uebrigen aber ist die Zunahme der weniger begünstigten Configurationen des Tolantetrachlorides mit steigender Temperatur durch diese Versuche bewiesen.

102. Ueber einige Derivate des Diphenylacetaldehyds;

von Wilhelm G. M. Weise.

Bei der Untersuchung der sogenannten Toluylenalkohole oder Hydrobenzoïne beobachteten Limpricht und Schwan er $\left.t^{* *}\right)$, dafs diese Körper sich beim Kochen mit verdünnter

*) Ber. d. deutsch. chem. Ges. I 1165.

**) Diese Annalen 160, 186. 